\title{
Should Antiarrhythmic Treatment Be Considered Doping in a Shooting Athlete?
}

Renata Rodrigues Teixeira de Castro ${ }^{1,2, *}$

\section{ORCID ID}

Castro RRT (D) https://orcid.org/0000-0001-5560-693X

\begin{abstract}
Beta-blockers are prohibited for athletes competing in particular sports. This article presents a case of sick sinus syndrome in a master shooting athlete and discuss the potential shooting accuracy enhancement obtained by the treatment beyond beta-blockade.
\end{abstract}

KEYWORDS: Doping; Beta-blocker; Shooting; Pacemaker; Performance.

\section{INTRODUCTION}

Doping refers to the use or intended use of prohibited substances and/or methods according to the Probibited List of the International Standard of the World Anti-Doping Agency, which is reviewed and updated at least once a year ${ }^{1}$. Prohibited drugs and methods are not the same for all sports. Whereas some athletes would benefit from increased power or speed, others may achieve better results through better steadiness and accuracy. This is the case of archery, shooting and other particular sports (Table 1), where the use of beta-blockers reduces heart rate and muscle tremor, consequently improving performance ${ }^{2}$. Although various drugs and even pacemakers can keep heart rate in a slow pace, only beta-blockers are considered doping.

One usually imagines that athletes are young and healthy, but this is not always true. While the average age of Olympic athletes in London Olympic Games (2012) was 26, there were 187 athletes over 40 years old in London Olympic Games. Most over-50s were competing in equestrian and shooting, and all of them were subject to doping control.

In fact, athletes are subjected to illnesses or conditions that require them to take particular medications. The oldest the athlete, greater are the chances that he/she will require a chronic medication use. If this medication

1. Universidade Iguaçu - Faculdade de Medicina - Nova Iguaçu/RJ - Brazil.

2. Cardiologia do Esporte - Rio de Janeiro/RJ - Brazil.

*Corresponding author: castrorrt@gmail.com

Received: Jul 24, 2020 | Accepted: Nov 17, 2020 
falls under the Prohibited List of the World Anti-Doping Agency ${ }^{1}$, a therapeutic use exemption (TUE) may give the athlete the authorization to take the medicine, as long as there is no other permitted treatment.

We present a case of a shooting athlete with sick-sinus syndrome and discuss the potential doping issue in this case.

Table 1. Sports where beta-blockers are prohibited in competition according to the World Antidoping Agency.

\begin{tabular}{lc}
\hline \multicolumn{1}{c}{ Sports where beta-blockers are prohibited in competition } & Also prohibited out-of-competition? \\
\hline Archery & Yes \\
Automobile & No \\
Billiards & No \\
Golf & Yes \\
Shooting & No \\
Skiing (jumping, freestyle aerials/halfpipe) & No \\
Snowboard (Halfpipe/big air) & No \\
Underwater sports in constant-weight apnea with or without fins, dynamic apnea \\
with and without fins, free immersion apnea, Jump Blue apnea, spearfishing, static \\
apnea, target shooting and variable weight apnea
\end{tabular}

\section{CASE REPORT}

A 57-year-old shooting amateur male athlete, with no previous comorbidities, was admitted to the hospital with recent onset palpitations and angina. The electrocardiogram revealed atrial fibrillation, and he received propafenone without success. Return to sinus rhythm was achieved after amiodarone. Coronary angiography was performed, and a myocardial bridge was found, without obstructive coronary lesions. A couple of months after hospital discharged, there was a new episode of atrial fibrillation, and cardioversion was successfully performed.

Three months later, the patient started to present presyncopal episodes, and a 24-hour Holter recording was done. The patient was not in use of any antiarrhythmic medication, but the recording showed moments of $2^{\text {nd }}$ degree atrioventricular block, mainly at night, with 95 sinus pauses (2.0 to 2.7 seconds each) and episodes of nonsustained ventricular tachycardia. Due to the sick sinus syndrome diagnosis, the medical decision was to implant a pacemaker, proceed with atrial fibrillation ablation and start regular diltiazem use. The patient remained asymptomatic for the next 3 months.

A cardiopulmonary exercise test was done. The patient started the exercise test with pacemaker-controlled rhythm which remained stable until the $3^{\text {rd }}$ minute of a ramp protocol when it was spontaneously substituted by sinus rhythm, with a normal chronotropic response during the test (heart rate reserve $=73 \%$ ).

The patient was cleared for practicing shooting and has recently won the gold medal in a local amateur championship. Local championships do not have doping control, but even if the patient had been selected for doping control, he would not be sanctioned, as there was no doping rule violation. But this case raised a question: Could the heart rate control by diltiazem and pacemaker have helped the patient to win the championship?

\section{DISCUSSION}

Drugs and methods are prohibited by the World Anti-Doping Agency when they meet at least two from the following criteria: if they enhance sport performance; if they would be harmful to athlete's health; or if they are against the spirit of sport. Almost all drugs included in the World Anti-doping Agency's Probibited List ${ }^{1}$ have the potential to treat some 
disease or medical condition. The fight for a clean competition should not deny legitimate medical treatment to an athlete. To overcome this, in 1992, the International Olympic Committee introduced a system known as therapeutic use exemption (TUE) $)^{3}$. This system allows athletes to administer prohibited drugs for genuine medical conditions and still compete. This authorization is granted by a TUE committee after confirming that: the athlete would experience significant health impairment if the medication was withheld; the prohibited substance would not increase the athlete's performance other than from restoring his/her health to normalcy; and, the athlete could not use a permitted alternative.

In the 1984 Olympic Games, in Los Angeles, the International Olympic Committee decided that athletes taking beta-blockers demanded a medical certificate to justify the use. Certificates were submitted by 18 athletes, who won 9 medals in shooting or modern pentathlon (which includes shooting as one of the five disciplines). Since 1985, the International Olympic Committee prohibited beta-blockers in sports in which performance could be enhanced by their use $\mathrm{u}^{3}$.

In 1987, Kruse at al. ${ }^{4}$ studied the effect of metoprolol on pistol shooting performance. Shooting improved $13.4 \%$ and the most skilled athletes demonstrated the clearest improvement with metoprolol. These authors found no correlation between the shooting improvement and changes in cardiovascular variables (changes of heart rate or systolic blood pressure). They have then suggested that the performance improvement was due to metoprolol central action of hand tremor reduction.

Since then, beta-blockers capable of crossing the blood-brain barrier are almost never approved as a TUE. Even cardioselective beta-blockers, which are believed to be less beneficial in reducing tremors than propranolol, are rarely allowed to be used.

The influence of beta-blockers on shooting accuracy are not exclusively related to tremor reduction, but also to heart rate control. The duration of systole and diastole change according to heart rate. During systole the whole body makes a small jerk that can cause brisk and small misalignment of the gun. Although really small, this misalignment is multiplied by the distance between the gun and the target affecting accuracy 5 .

Gallicchio et al. ${ }^{6}$ examined the relationship between shooting accuracy and the phase of the cardiac cycle in which the shot is fired. The researchers recorded shooting accuracy and timing of each shot relative to electrocardiogram R-waves. They found that the phase of cardiac cycle when shot is fired affects shooting accuracy and that shooters should avoid firing at pressure pulse wave peak. Thus, best shooting performance could be achieved when athletes are trained to fire during diastole $^{5}$. As these results would be easier achieved with low heart rates than with higher ones, elite shooters usually try to reduce heart rate before shooting.

Shooter's coaches believe that biofeedback techniques can improve shooter ability to sense heart beats and trigger during diastole ${ }^{7,8}$. As the only banned class of drugs that slow heart rate are beta-blockers, athletes may potentially improve shooting accuracy through the use of calcium-channel blockers, amiodarone or digoxin, which are not include in World Anti-Doping Agency's Prohibited List. The potential benefit of heart rate controlling to achieve better shooting performance could even be achieved by pacemaker, as in the reported case.

The mental stress of shooting and competing can increase shooter's heart rate. As heart rate increase may affect shooting accuracy, this is a physiologic, although undesired, response. Pacemakers rate adaptative sensors are intended to simulate normal sinus response. An ideal sensor should be able to detect exercise and nonexercise related demands in a specific way, avoiding nonphysiologic changes ${ }^{9,10}$. Accelerometer pacing mode can detect patient's movements and increase heart rate accordingly to exercise demands, but this would not happen in situations with little or absence of movements. Pacemakers with closed loop stimulation sensors algorithm responds with appropriate heart rate responses to acute mental stress. Thus, pacemaker sensor algorithm may be relevant to shooters.

The World Antidoping Agency respects medicine best practices. Thus, in most cases where there is no other therapeutic alternative, the World Antidoping Agency allows the use of beta-blockers, so that the athlete can return to a state of normal health. Nevertheless, it seems that beta-blockers, other drugs with chronotropic negative effect and even pacemakers with different algorithms may provide additional enhancement of performance in shooters beyond what might be anticipated by the athlete's return to a state of normal health. The analysis of each case, taking into consideration medical and sports ethics is a future challenge for the World Antidoping Agency. 


\section{CONCLUSIONS}

Physicians must be aware of the potential performance enhancement potential of medications when treating athletes. Beta-blockers, other with chronotropic negative effect and methods that control heart rate may enhance shooting accuracy, but only beta-blockers are prohibited substances in accuracy sports.

\section{REFERENCES}

1. [WADA] World Anti-Doping Agency (2018). Prohibited List: January 2019. The World Anti-Doping Code. International Standard. Montreal: WADA.

2. Davis E, Loiacono R, Summers RJ. The rush to adrenaline: drugs in sport acting on the beta-adrenergic system. Br J Pharmacol. 2008;154(3):584-97. https://doi.org/10.1038/bjp.2008.164

3. Fitch K. Proscribed drugs at the Olympic Games: permitted use and misuse (doping) by athletes. Clin Med (Lond). 2012;12(3):25760. https://doi.org/10.7861/clinmedicine.12-3-257

4. Kruse P, Ladefoged J, Nielsen U, Paulev PE, Sørensen JP. Beta-blockade used in precision sports: effect on pistol shooting performance. J Appl Physiol. 1986;61(2):417-20. https://doi.org/10.1152/jappl.1986.61.2.417

5. Helin $\mathrm{P}$, Sihvonen $\mathrm{T}$, Hänninen $\mathrm{O}$. Timing of the triggering action of shooting in relation to the cardiac cycle. Br J Sports Med. 1987;21(1):33-6. https://doi.org/10.1136/bjsm.21.1.33

6. Gallicchio G, Finkenzeller T, Sattlecker G, Lindinger S, Hoeldmoser K. The influence of physical exercise on the relation between the phase of cardiac cycle and shooting accuracy in biathlon. Eur J Sport Sci. 2019;19(5):567-75. https://doi.org/10.1080/17461391.2 018.1535626

7. Mullineaux DR, Underwood SM, Shapiro R, Hall JW. Real-time biomechanical biofeedback effects on top-level rifle shooters. Appl Ergon. 2012;43(1):109-14. https://doi.org/10.1016/j.apergo.2011.04.003

8. Ortega E, Wang CJK. Pre-performance physiological state: heart rate variability as a predictor of shooting performance. Appl Psychophysiol Biofeedback. 2018;43(1):75-85. https://doi.org/10.1007/s10484-017-9386-9

9. Alt E, Matula M, Theres H, Heinz M. Principle of the activity-controlled rate-adaptive cardiac pacemaker: analysis of stress and environment-induced mechanical effects on the human body. Z Kardiol. 1989;78(9):587-97.

10. Charles RG, Heemels JP, Westrum BL. Accelerometer-based adaptive-rate pacing: a multicenter study. European EXCEL Study Group. Pacing Clin Electrophysiol. 1993;16(3 Pt 1):418-25. https://doi.org/1 0.1111/j.1540-8159.1993.tb01604.x 